Article

\title{
On the Luminescence Properties and Surface Passivation Mechanism of III- and N-Polar Nanopillar Ultraviolet Multiple-Quantum-Well Light Emitting Diodes
}

\author{
Moheb Sheikhi ${ }^{1,2,+}$, Yijun Dai ${ }^{1,2,+}$, Mei Cui ${ }^{1,2}$, Liang Li ${ }^{1,2}$, Jianzhe Liu ${ }^{3}$, Wenan Lan ${ }^{3}$, \\ Rongrong Jiang ${ }^{1}$, Wei Guo ${ }^{1,2, * \mathbb{D}}$, Kuan W.A. Chee ${ }^{4,5, *}$ and Jichun Ye ${ }^{1,2}$ \\ 1 Ningbo Institute of Materials Technology and Engineering, Chinese Academy of Sciences, \\ Ningbo 315201, China; moheb@nimte.ac.cn (M.S.); daiyijun@nimte.ac.cn (Y.D.); cuimei@nimte.ac.cn (M.C.); \\ lliang@nimte.ac.cn (L.L.); jiangrr@nimte.ac.cn (R.J.); jichun.ye@nimte.ac.cn (J.Y.) \\ 2 University of Chinese Academy of Sciences, Beijing 100049, China \\ 3 Zhe Jiang Bright Semiconductor Technology Co., Ltd., Jinhua 321016, China; jzliu@bst-group.cn (J.L.); \\ ben@bst-group.cn (W.L.) \\ 4 Hefei National Laboratory for Physical Sciences at Microscale, and Department of Physics, \\ University of Science and Technology of China, Hefei 230026, China \\ 5 Laser Research Institute, Shandong Academy of Sciences, Qingdao 226100, China \\ * Correspondence: guowei@nimte.ac.cn (W.G.); kuan.chee@cantab.net (K.W.A.C.) \\ $\dagger$ These authors contribute equally to this work.
}

Received: 6 November 2019; Accepted: 17 December 2019; Published: 5 June 2020

\begin{abstract}
The non-centrosymmetricity of III-nitride wurtzite crystals enables metal or nitrogen polarity with dramatically different surface energies and optical properties. In this work, III-polar and N-polar nanostructured ultraviolet multiple quantum wells (UV-MQWs) were fabricated by nanosphere lithography and reactive ion etching. The influence of $\mathrm{KOH}$ etching and rapid thermal annealing treatments on the luminescence behaviors were carefully investigated, showing a maximum enhancement factor of 2.4 in emission intensity for III-polar nanopillars, but no significant improvement for N-polar nanopillars. The discrepancy in optical behaviors between III- and N-polar nanopillar MQWs stems from carrier localization in III-polar surface, as indium compositional inhomogeneity is discovered by cathodoluminescence mapping, and a defect-insensitive emission property is observed. Therefore, non-radiative recombination centers such as threading dislocations or point defects are unlikely to influence the optical property even after post-fabrication surface treatment. This work lays solid foundation for future study on the effects of surface treatment on III- and N-polar nanostructured light-emitting-diodes and provides a promising route for the design of nanostructure photonic devices.
\end{abstract}

Keywords: III-nitride thin film; nanostructures; ultraviolet emitters; surface passivation; luminescence intensity

\section{Introduction}

III-nitride based ultraviolet light-emitting-diodes (UV-LEDs) are useful for many applications including UV curing, photo therapy and UV disinfection, due to the direct bandgap property of wurtzite phase III-nitride crystals [1-4]. However, the extraction efficiency of UV LEDs is relatively low due to the strong internal light reflection and strong transverse magnetic (TM) polarization of the emitted light [5-7]. Challenges in light extraction have encouraged research and development of small length scale devices for new applications, like nanostructured LEDs, for example [8-10]. The three-dimensional geometry of the nanopillars or nanoholes allows light to be extracted from the 
sidewalls of the nanostructures. This becomes increasingly important as the emission wavelength of the LEDs moves from visible to ultraviolet wavelength region due to increasing proportion of TM-polarized light, as well as the growing impact of light absorption in the p-AlGaN contact layer [11,12]. Additionally, the benefits from strain relaxation in nanostructured LEDs means reduced influence from quantum confined stark effect (QCSE), which has an adverse impact on the electron and hole wavefunction overlap [13].

Currently, most of the nanostructured UV-LEDs are fabricated by reactive ion etching (RIE) due to better control over morphologies and sizes of the nanostructures [14]. However, RIE introduces surface defect states that will lead to reduced external quantum efficiency (EQE) and degraded output power of LEDs through Shockley-Read-Hall (SRH) non-radiative recombination [15]. The large surface areas of GaN nanopillars can lead to dangling bonds during nanopillar formation. However, the resulting surface defects can be reduced by passivation techniques such as chemical etching or dielectric deposition [16]. It was reported that, plasma-related damages at the sidewalls of multiple quantum wells (MQWs) induced by RIE can be readily healed by thermal annealing, reducing the density of surface traps [17]. Therefore, eliminating the surface defect states is strongly required [18]. Chiu et al. reported that after a photo-enhanced chemical (PEC) wet oxidation process, emission from InGaN/GaN-based random nanorod LEDs was significantly improved, but also with a 10.5-nm blueshift with respect to the as-grown LED [19]. Sun et al. also reported a 50\% enhancement in the UV light emission intensity from the $\mathrm{KOH}$ treated InGaN nanowires (NWs) due to the removal of surface dangling bonds [18]. Despite the above promising results, the knowledge regards to surface passivation of nanostructured LED is still not complete, since the majority of the samples are III-polar for better crystalline quality and smoother surfaces. However, N-polar LEDs have their unique advantage such as lower contact resistance, increased current injection efficiency, and an internal polarization field, which is opposite to the external bias, leading to reduced QCSE and higher radiative recombination rate $[20,21]$. Unfortunately, there seldom are comparative investigations on the effects of surface treatments on the optical properties of both III- and N-polar nanostructured MQWs LEDs, which serve as building blocks of next-generation high-efficiency UV emitters.

In this work, large-scale, highly periodic nanopillar UV MQWs with III- and N-polarities were fabricated by nanosphere lithography and RIE patterning. Chemical treatment and thermal annealing are acknowledged as promising methods to enhance the luminescence property of III-polar nanostructured MQWs by 2.4 times at most, but was demonstrated to show little effect on N-polar nanostructures. The underlying reason for this discrepancy was thoroughly discuss, which can be mainly attributed to intrinsic indium localization and herein defect-insensitive emissions from the $\mathrm{N}$-polar nanostructures. A comprehensive investigation on the surface passivation mechanism on both III- and N-polar nanostructure emitters were provided, benefiting future development of novel nanostructured light emitters.

\section{Materials and Methods}

InGaN/GaN based UV-MQW was grown on 2-inch c-plane sapphire substrate via a low-pressure, high-temperature metalorganic chemical vapor deposition (MOCVD) system. Trimethylindium (TMIn), triethylgallium (TEGa) and ammonia $\left(\mathrm{NH}_{3}\right)$ were used as precursors of $\mathrm{In}, \mathrm{Ga}$ and $\mathrm{N}$, respectively. Hydrogen $\left(\mathrm{H}_{2}\right)$ was used as the carrier gas. To investigate the polarity influence on the luminescence property of nanopillars, both III- and N-polar planar MQWs were firstly grown. The III-nitride polarity of the MQWs was controlled by the modulation of low-temperature (LT) AlN nucleation layer (NL) prior to high-temperature (HT) epitaxial growth. Generally, III-nitride thin film is III-polarity if it is grown on LT AlN-NL. In contrast, when grown on bare sapphire substrate with proper $\mathrm{H}_{2}$ annealing and $\mathrm{NH}_{3}$ nitridation condition, the thin film is N-polarity. UV-MQW consists of a $3.5 \mu \mathrm{m}$ GaN epitaxial layer, eight pairs of $\operatorname{In}_{0.03} \mathrm{Ga}_{0.97} \mathrm{~N} / \mathrm{GaN}$ MQWs followed by a $10 \mathrm{~nm}$ GaN cap layer. To fabricate nanostructured UV-MQWs, III-polar and N-polar epitaxial thin films are uniformly coated with polystyrene (PS) spheres of $2 \mu \mathrm{m}$ diameter via a large-area micro-propulsive injection method. 
The colloidal solution was injected into the water to form a Langmuir-Blodgett film in a hexagonal configuration at the air/water interface. By draining away the water, the monolayer of closely packed PS spheres is transferred onto the MQW thin film. Nanopillar arrays were then achieved by RIE using $\mathrm{Cl}_{2} / \mathrm{BCl}_{3}$ gases to pattern transfer the PS spheres to the underlying MQWs. The remaining PS spheres were removed by sonication in acetone solution. After fabrication of the nanopillar arrays, chemical and thermal treatments were applied to the MQW samples in order to heal the plasma-related damages. For chemical treatment, samples were dipped into $\mathrm{KOH}$ aqueous solution with a concentration of $10 \mathrm{wt} \%$. The treatment was performed under room temperature (RT) or $45^{\circ} \mathrm{C}$ for $40 \mathrm{~s}$. For thermal treatment, rapid thermal annealing (RTA) was performed at $800{ }^{\circ} \mathrm{C}$ or $900{ }^{\circ} \mathrm{C}$ for 15 min under $\mathrm{N}_{2}$ atmosphere. Surface morphologies of the nanostructures were characterized in the Hitachi S-4800 field-emission (FE) SEM (Hitachi, Tokyo, Japan). Dislocation densities of both samples were characterized using a point-focused high-resolution X-ray $(\mathrm{Cu} \mathrm{K} \alpha 1)$ diffractometer $(\mathrm{HRXRD \text {, }}$ Bruker D8 Discover, Germany) equipped with a four-bounce symmetric Ge (220) monochromator. The surface morphology of the MQW structure was characterized by atomic force microscopy (AFM) (Veeco Dimension 3100 V, Plainview, NY, USA). High-angle annular dark field scanning transmission electron microscopy (HAADF-STEM) was performed and weak beam dark field images were acquired under two beam conditions at an acceleration voltage of $300 \mathrm{kV}$ (FEI Titan ST microscope, Hillsboro, OR, USA). Specimens were prepared by focus ion beam (FIB) using an FEI Helios SEM system with a Ga ion source. RT photoluminescence (PL) was performed by using an Ar-F (193 nm) excimer laser (Coherent Inc., Santa Clara, CA, USA) as an excitation pumping source, and the spectra were collected by a Horiba iHR550 spectrometer (Horiba, Kyoto, Japan). Cathodoluminescence (CL) investigations were undertaken using a Horiba MP 325 CL characterization system (Horiba, Kyoto, Japan) with voltage of $5 \mathrm{kV}$ and current level of $188 \mu \mathrm{A}$. Strain conditions of the nanopillar MQWs were investigated by a Renishaw inVia Reflex spectrometer system (Renishaw, New Mills, UK) with a $532 \mathrm{~nm}$ Nd-YAG laser as the excitation source. The surface stoichiometry of the samples was further studied by Kratos Axis Ultra DLD X-ray photoelectron spectrometer (XPS; Kratos, Manchester, UK).

\section{Results and Discussion}

Before detailed investigations on optical properties of nanopillar samples, the structural and surface morphology information of planar III-polar and N-polar MQW samples are investigated. Figure 1 shows the HRXRD rocking curve (RC) scans of both III- and N-polar samples. The full-width-half-maximum (FWHM) values of RC scans were used to evaluate the crystalline quality of III-nitride thin films. From the fitting, the FWHM of (002) and (102) peaks of III-polar sample are 265 and 275 arcsec, respectively, which are lower than those of N-polar samples (586 and 578 arcsec for (002) and (102) peaks). The dislocation density can herein be estimated. Screw and edge type dislocation densities are $1.53 \times 10^{8} \mathrm{~cm}^{-2}$ and $4.59 \times 10^{8} \mathrm{~cm}^{-2}$ for III-polar samples, which are approximately 5 times lower than those of N-polar ones, whose values are $7.48 \times 10^{8} \mathrm{~cm}^{-2}$ and $1.8 \times 10^{9} \mathrm{~cm}^{-2}$. This result suggests that III-polar samples exhibit superior crystalline quality than N-polar samples.

Surface morphologies of both samples were analyzed by AFM and illustrated in Figure 2. Clear bi-layer steps are illustrated in the III-polar sample, demonstrating a typical step-flow growth mode of III-polar III-nitride thin film [22]. On the other hand, N-polar sample is covered with hillocks of a few micrometers lateral size, suggesting a three-dimensional growth mode because of its much smaller surface energy and thus larger nucleation density [23]. Root-mean-square roughness for IIIand N-polar samples are 0.37 and $11.0 \mathrm{~nm}$, respectively. The drastic difference of surface roughness of these two polarities are also correlated with their crystalline quality. Indium content of planar MQW samples can usually be identified from symmetric and asymmetric $\omega-2 \theta$ scans from HRXRD. Symmetric $\omega-2 \theta$ scan of planar III- and N-polar samples was performed and shown in supplementary Figure S1. The most prominent peak can be identified as GaN template. Different peak positions originate from various strain states inside epitaxial thin films as a consequence of growth mode difference between III- and N-polar domains [21]. Usually, 0st order InGaN MQW peak can be found 
on the low-angle side of the spectrum, but can hardly be found in our study. This could possibly due to the extremely low in content of only 3\% in the MQW region and thus MQW peak merges with the GaN template peak.
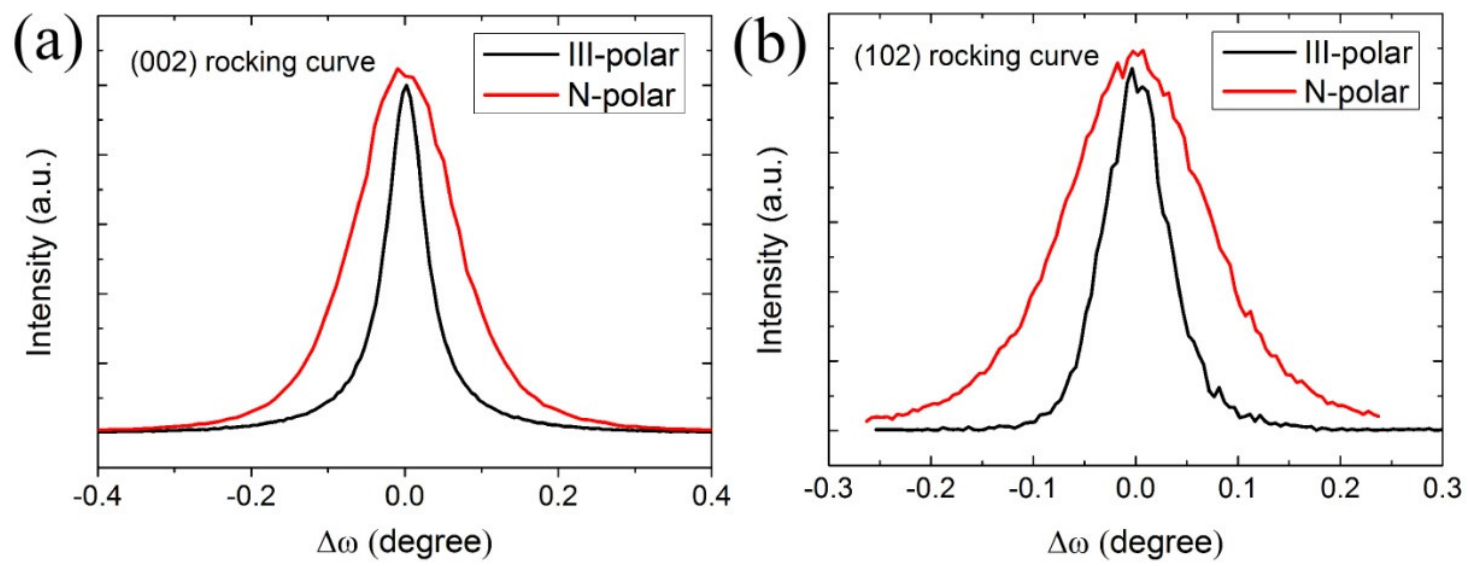

Figure 1. HRXRD (002) (a) and (102) (b) RC scans of planar III- and N-polar samples for dislocation estimation.
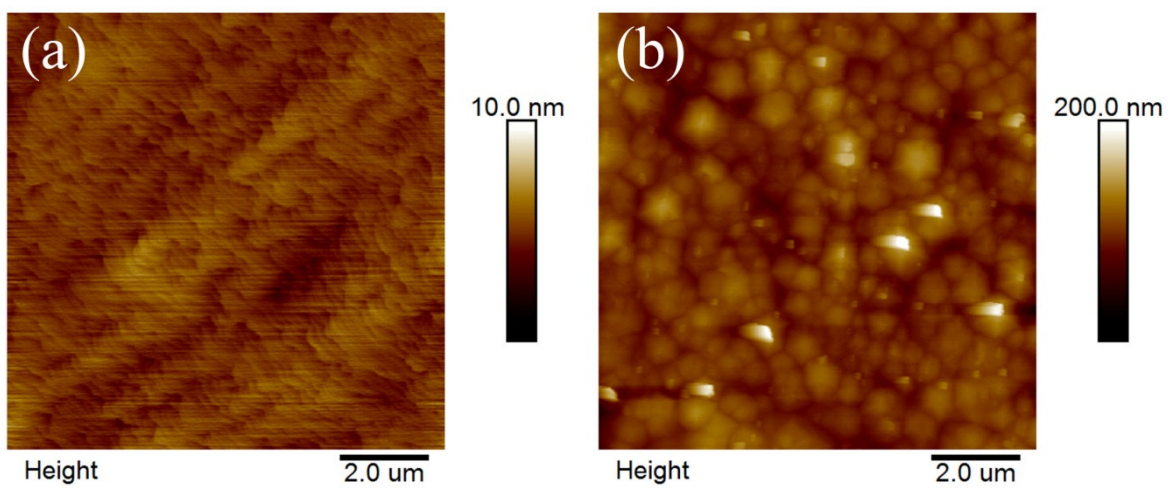

Figure 2. AFM surface morphology of planar III-polar (a) and N-polar (b) MQW samples.

RIE followed by nanosphere lithography was utilized to fabricate the nanopillar UV-MQW samples. The lattice constant of the nanopillar array is expected to match the $2 \mu \mathrm{m}$ diameter of the PS spheres used in the nanosphere lithography. Due to ion bombardment, amorphous layers are generated on the sidewall surfaces of the nanopillars during RIE patterning, but which are expected to be removed by the $\mathrm{KOH}$ treatment. The SEM images in Figure 3 show the surface morphology of the uniformly distributed nanopillar hexagonal array on the as-fabricated III- and N-polar UV-MQWs after subjecting to the different treatments. It can be seen that the diameter of the nanopillars remained the same after RTA but reduced slightly to $1.98 \mu \mathrm{m}$ after $\mathrm{KOH}$ treatment. Thermal annealing does not alter the surface morphologies of the nanopillars due to a lacking of energy for thin film re-crystallization or surface reconstruction. It is known that the dangling bond density for N-polar planes is higher than that of (0001) planes, leading to a much higher etch rate under $\mathrm{KOH}$ treatment. Therefore, elongated time of $\mathrm{KOH}$ etching could damage the nanopillars as shown in Figure 3e where the flat top mesa of the nanopillars is covered with small hillocks. The $60^{\circ}$ tilted view SEM images of the as-fabricated nanopillars are shown in the inset of Figure 3a,d. After RIE process, the depths of the III and N-polar nanopillars are 0.744 and $0.748 \mu \mathrm{m}$, respectively, suggesting almost same RIE etching rate between these two polarities. 

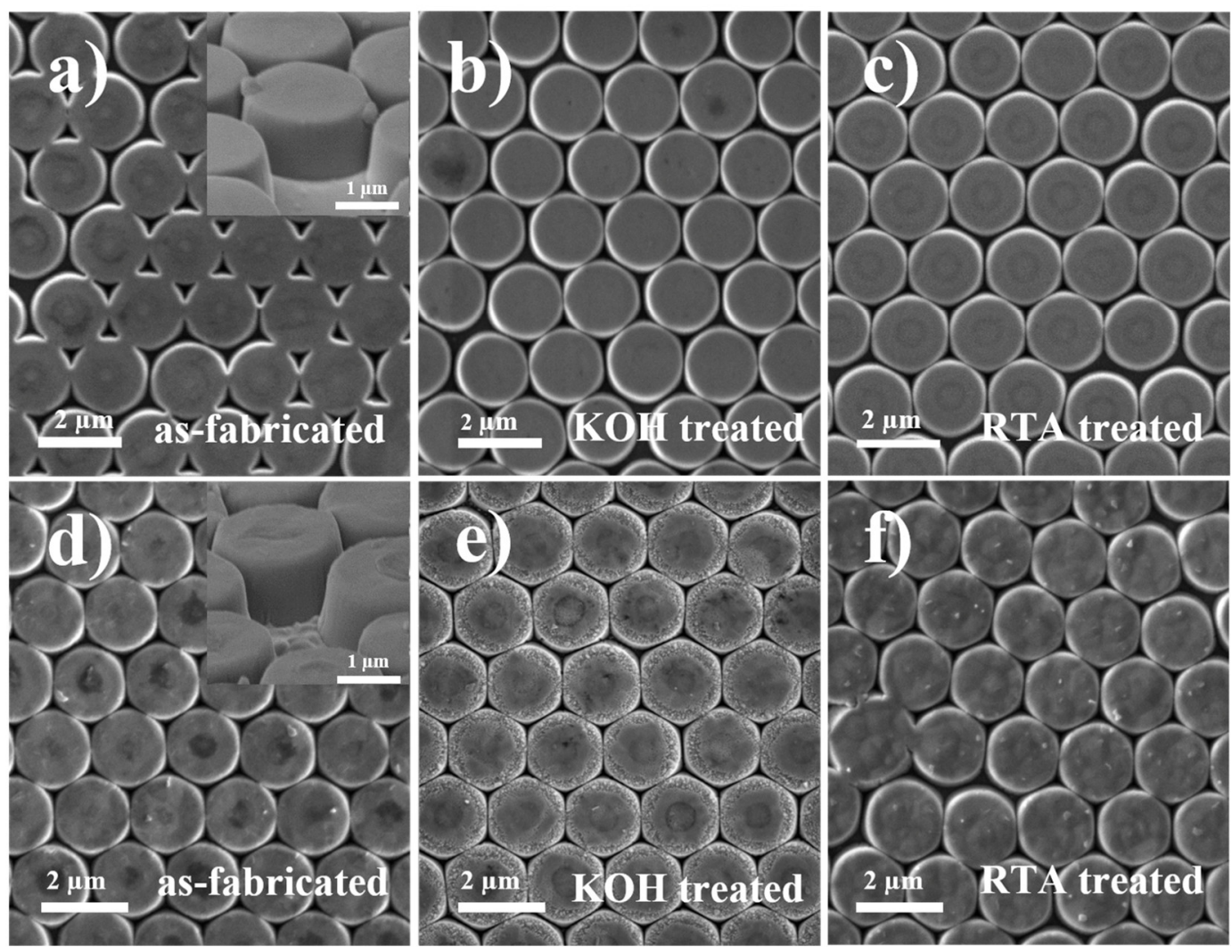

Figure 3. SEM images of III-polar (a-c) and N-polar (d-f) nanopillar multiple quantum wells (MQWs) before and after the $\mathrm{KOH}$ or rapid thermal annealing (RTA) treatment. Tilted-view SEM images of as-fabricated nanopillars are shown in the inset of $(\mathbf{a}, \mathbf{d})$.

RT photoluminescence (PL) measurements were carried out to determine the luminescence efficiency of the nanopillar MQWs before and after $\mathrm{KOH}$ or RTA treatment. First of all, PL intensities of III- and N-polar nanostructured samples are compared. III-polar nanopillars show stronger intensity than that of N-polar samples. This could because of two reasons: firstly, a strong carrier localization effect is observed in N-polar nanopillar sample as will be demonstrated later. This could originate from rough surface morphology of N-polar planar sample to begin with, and MQW thickness fluctuations as demonstrated in our previous work [20]. Even though carrier localization can greatly enhance internal quantum efficiency in LEDs, but excessive phase separation can also lead to a greatly reduced active area of the MQWs at the same time, which is detrimental to the optical property as observed in this work. Secondly, N-polar sample exhibits higher dislocations than that of III-polar samples. This greatly deteriorate the luminescence intensity of N-polar nanostructured samples. PL spectra of planar III- and N-polar MQW samples are shown in supplementary Figure S2 for comparison purpose. No obvious variation in peak position is observed between planar and nanostructured sample. However, an enhanced luminescence intensity is identified for the nanostructured sample due to enhanced light scattering effect [14].

PL spectra subject to different surface treatments were further investigated. Figure $4 a, b$ shows the PL spectra of III- and N-polar nanopillar MQWs after the respective treatments. PL intensities dramatically increased for III-polar compared to N-polar nanopillar MQWs. The highest PL enhancement reached 2.4 times for RTA of $900{ }^{\circ} \mathrm{C}$. This can be well explained by the passivation of surface traps and the healing of surface-related defects [14]. However, the luminescence spectra were dramatically different for N-polar nanopillar MQWs. For the N-polar nanopillar MQW, a peak and a broad shoulder were identified in the PL spectra where the left peak was located at approximately $386 \mathrm{~nm}$, and the right shoulder was located at $420 \mathrm{~nm}$. The left peak intensity remained roughly the same after $\mathrm{KOH}$ or RTA treatment, while the longer wavelength shoulder reduced, suggesting that 
polarity played a critical role in the influence of surface treatment on luminescence. The integrated PL intensities of the III- and N-polar nanopillars are shown in Figure 4c, indicating that the effect of surface treatment was marginal on the N-polar MQWs compared to that of the III-polar samples.
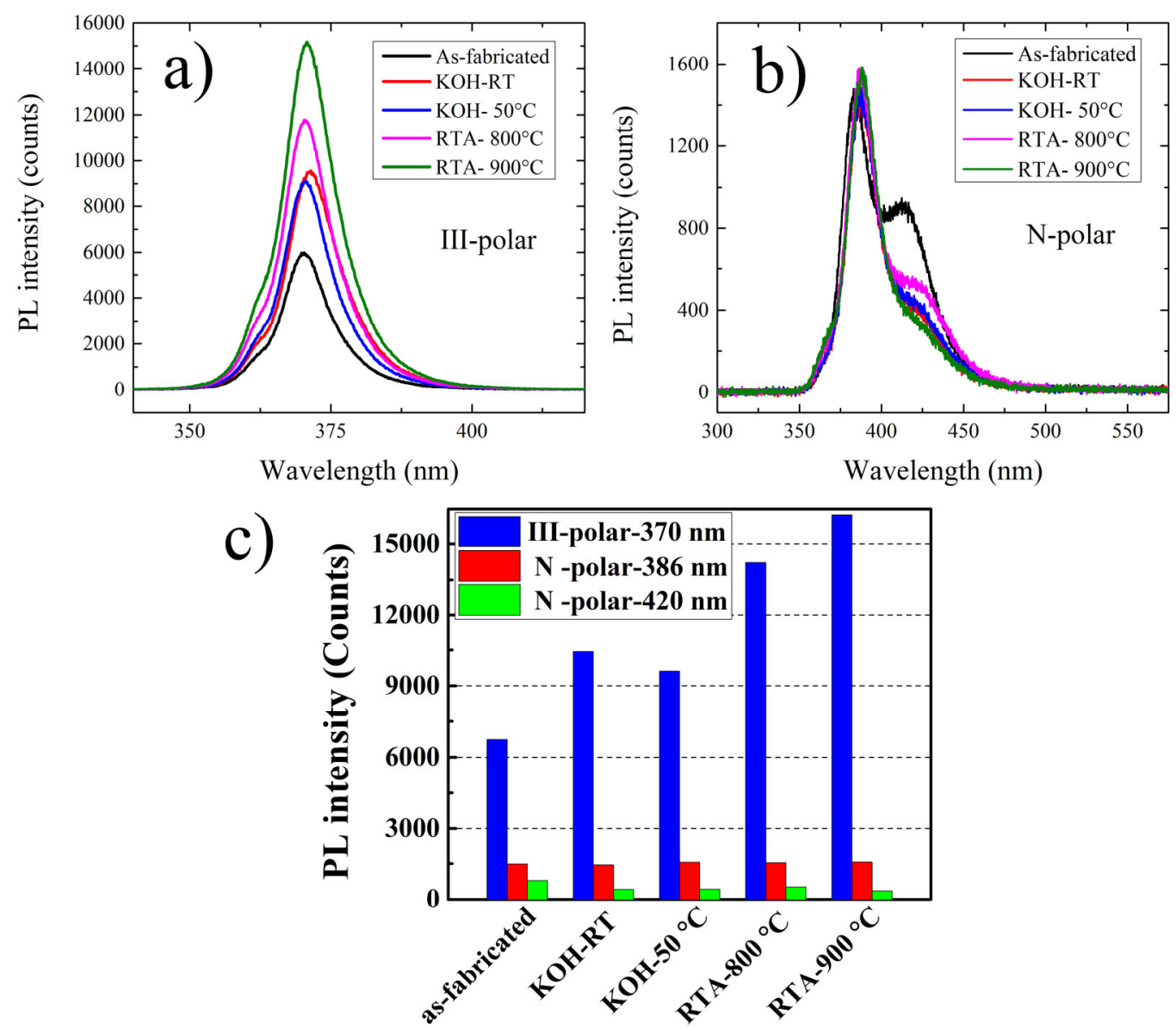

Figure 4. Room temperature (RT) photoluminescence (PL) spectra of III-polar (a) and N-polar (b) nanopillar MQWs, and integrated PL intensity of III-polar and N-polar MQW nanopillar MQWs at the specific peak wavelengths (c), before and after $\mathrm{KOH}$ treatment or rapid thermal annealing (RTA).

The strong variation of PL peak position from III- and N-polar samples is originated from either higher indium composition or thickness variation or a combination of both. An average indium content changing from $1.3 \%$ to $5.2 \%$ in the MQW is expected when composition is considered as the only factor. For the $420 \mathrm{~nm}$ shoulder peak in N-polar MQW sample, a further increase of indium content to $12.6 \%$ is obtained. The optical transition in the QWs was further analyzed by solving the Poisson equations and carrier transport equations. During calculation, 3/11 $\mathrm{nm} \operatorname{In}_{0.03} \mathrm{Ga}_{0.97} \mathrm{~N}$ QW and GaN QB thicknesses are utilized. As shown in the calculation result illustrated in supplementary Figure S3, a broad emission peak with position located at $365 \mathrm{~nm}$ is identified, which is slightly shorter than the $370 \mathrm{~nm}$ emission peak observed in PL spectra. The internal electric field inside QW is less than $1 \times 10^{5} \mathrm{~V} / \mathrm{cm}$, which is relatively low compared to AlGaN or InGaN system reported elsewhere [24,25]. The discrepancy between the simulated electroluminescence (EL) spectrum and experimental PL spectrum can be explained by a variety of factors including different interaction volumes between injected current in EL and excited photons in PL, deviation of indium composition from target and QW thickness fluctuation. But we can safely conclude that the uniform emission peak from III-polar sample is a consequence of efficient radiative recombination inside the abrupt QWs as shown from STEM image of III-polar MQW sample observed in supplementary Figure S4, while non-uniform emission in $\mathrm{N}$-polar sample is a result of carrier localization. 
Finally, in order to correlate PL spectra with the influence of QW thickness variation, emission spectra are simulated based on an LED with 3 pairs of MQWs. The indium content in QW is fixed at $3 \%$, while QW thickness was varied from $3 \mathrm{~nm}$ to $6 \mathrm{~nm}$. The thickness of quantum barrier remains constant at $11 \mathrm{~nm}$. From the simulation results shown in supplementary Figure S5, two emission peaks can be identified. This occurrence of two peaks can be explained by the shallow QW and thus weak carrier confinement. The high-energy emission peak approximately locates at $364 \mathrm{~nm}$, and does not change with QW thickness. On the other hand, the low-energy peak red shifts as increasing QW thickness, in good agreement with the reduced quantum confinement and thus lower emission energy [26]. However, the red shift is only less than $7 \mathrm{~nm}$, which is far less than that observed in the experiment. The coherence with a 420-nm emission cannot be simulated by only changing the QW thickness, which further demonstrates that composition inhomogeneity must play an important role in the light emission.

To further understand the PL enhancement mechanism of III-polar nanopillar MQWs, PL efficiency $\left(\mathrm{PL}_{\mathrm{eff}}\right)$ as a function of excited power density of the III and N-polar MQW samples is illustrated in Figure 5. PL $\mathrm{P}_{\mathrm{eff}}$ is defined as the ratio of PL intensity to excited power density, and its slope is an indication of radiative recombination mechanism [10]. The negative slope of the PL $\mathrm{L}_{\mathrm{eff}}$ versus excited power density for planar MQWs suggests the existence of exciton-related emission together with large amount of non-radiative centers. In contrast, for III-polar nanopillar samples, the PL $\mathrm{eff}_{\text {increased }}$ when the pumping power density increased, which is a signature of radiative carrier recombination through free carriers. The $\mathrm{PL}_{\mathrm{eff}}$ increased for $\mathrm{KOH}$ treatment at a higher temperature. Higher $\mathrm{PL}_{\mathrm{eff}} \mathrm{Was}$ obtained after thermal annealing. The slightly larger slope of $\mathrm{PL}_{\mathrm{eff}} \mathrm{vs}$. pumping power density after thermal annealing shows an enhanced radiative recombination rate, which might indicate annihilation of trap defects. For N-polar nanopillar MQWs, since the emission spectra had two peaks locating at $386 \mathrm{~nm}$ and $420 \mathrm{~nm}$, thus two $\mathrm{PL}_{\text {eff }}$ curves are illustrated. For a $386 \mathrm{~nm}$ emission in N-polar MQW, $\mathrm{PL}_{\mathrm{eff}}$ also increased with pumping power density similar to that of III-polar nanopillars. However, a negative slope was observed for $\mathrm{N}$-polar nanopillars with $420 \mathrm{~nm}$ emission, again suggesting the exciton-related emission mechanism, which was a signature of carrier localization effect as will be discussed in details later in Figures 6 and 7.

Due to phase separation between In and Ga, compositional inhomogeneities in InGaN alloys lead to non-uniform optical properties of UV-LEDs, especially for N-polar surfaces where rough surface morphology promotes phase separation. Therefore, deeper understanding on the spatially resolved luminescence properties of III- and N-polar nanopillar MQWs is critically important. Panchromatic CL intensity mapping is shown in Figure 6. Figure 6a-d illustrates the CL distribution in III-polar nanopillar MQWs and Figure 6e-h illustrates the CL distribution in N-polar nanopillar MQWs. Under low magnification, bright stripes along the substrate offcut direction were clearly observed on the surface of the III-polar nanopillar MQW (Figure 6a), which could be attributed to intrinsic indium localization in the MQWs. The strong luminescence comes from the In-rich clusters along the step edges of the epitaxial thin films. Since white stripes are shown in both planar and nanostructured MQWs, nanopillar fabrication did not have an influence on the distribution of luminescence centers. A closer look at the spatially resolved CL intensity distribution of III-polar MQWs in Figure 6b-d gives us clear information that luminescence only coming from the nanopillars where MQWs were not etched away by RIE. Additionally, the emission intensity increased dramatically after thermal annealing of $900{ }^{\circ} \mathrm{C}$, which agreed perfectly with the enhanced PL intensity as shown before in Figure 4. Different from that of III-polar samples, strong localization of luminescence centers is shown for all N-polar samples. The dot-like bright features have lateral size of 20-200 nm. White dots represent quantum-dot-like luminescence centers, which were uniformly distributed on the sample, possibly due to the rough surface morphology of N-polar MQWs and thus non-uniform light emission. Note that carrier localization is also observed in planar samples. This has been thoroughly discussed in our previous work $[27,28]$. Carrier localization was observed in N-polar domain of planar sample due to thickness and composition fluctuation. However, carrier localization is not observed in III-polar MQW sample. 
This is due to the fact that the indium composition is too low for spinodal decomposition. In fact, carrier localization was widely reported in InGaN with high indium content above 15\% [29], but seldom been reported in UV-LEDs with InGaN MQWs of indium content less than 5\% as studied here.
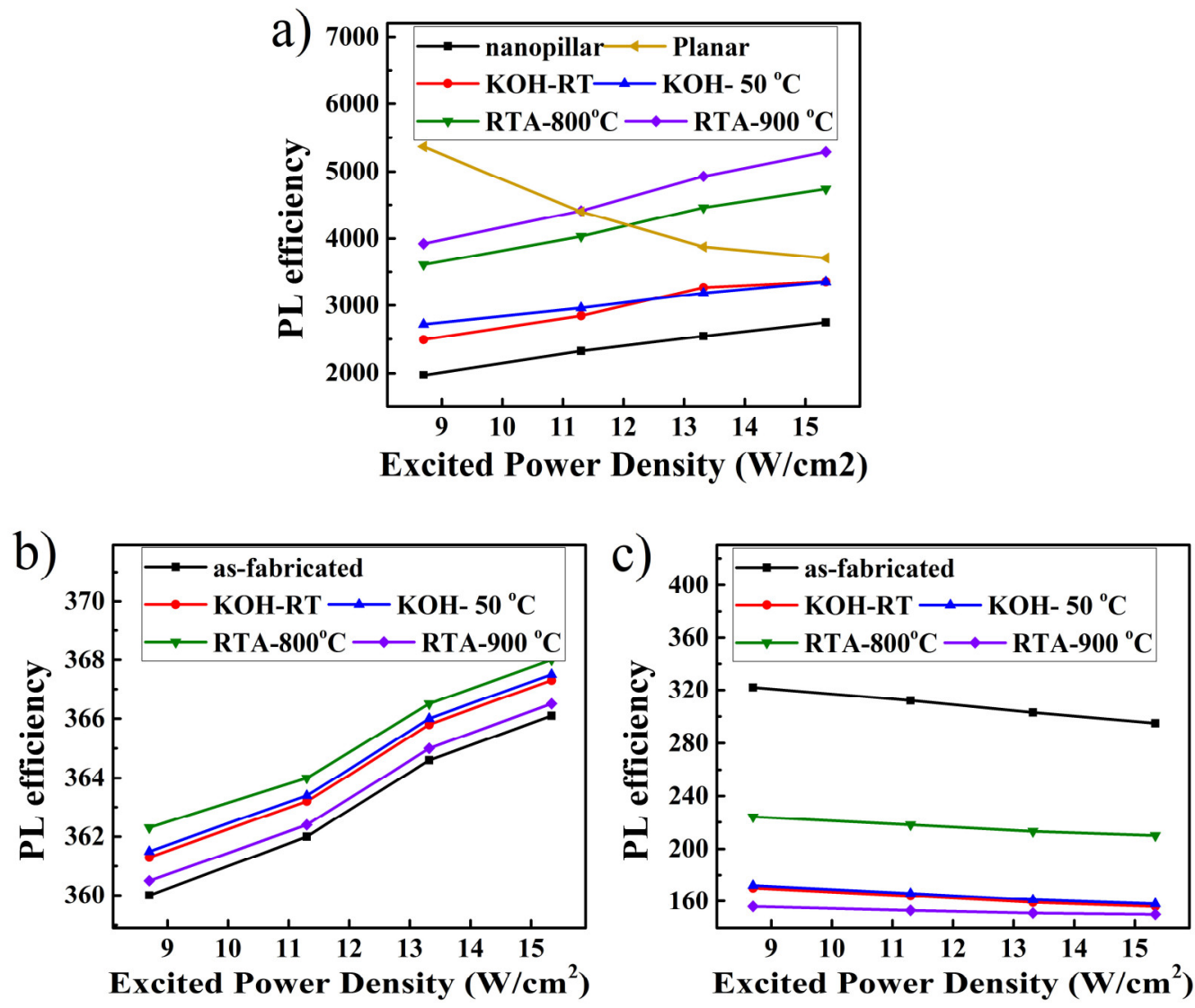

Figure 5. PL efficiency as a function of excited power density for III-polar nanopillar MQWs (a), and N-polar nanopillar MQWs with emission wavelength located at $386 \mathrm{~nm}$ (b) and at $420 \mathrm{~nm}$ (c) respectively.
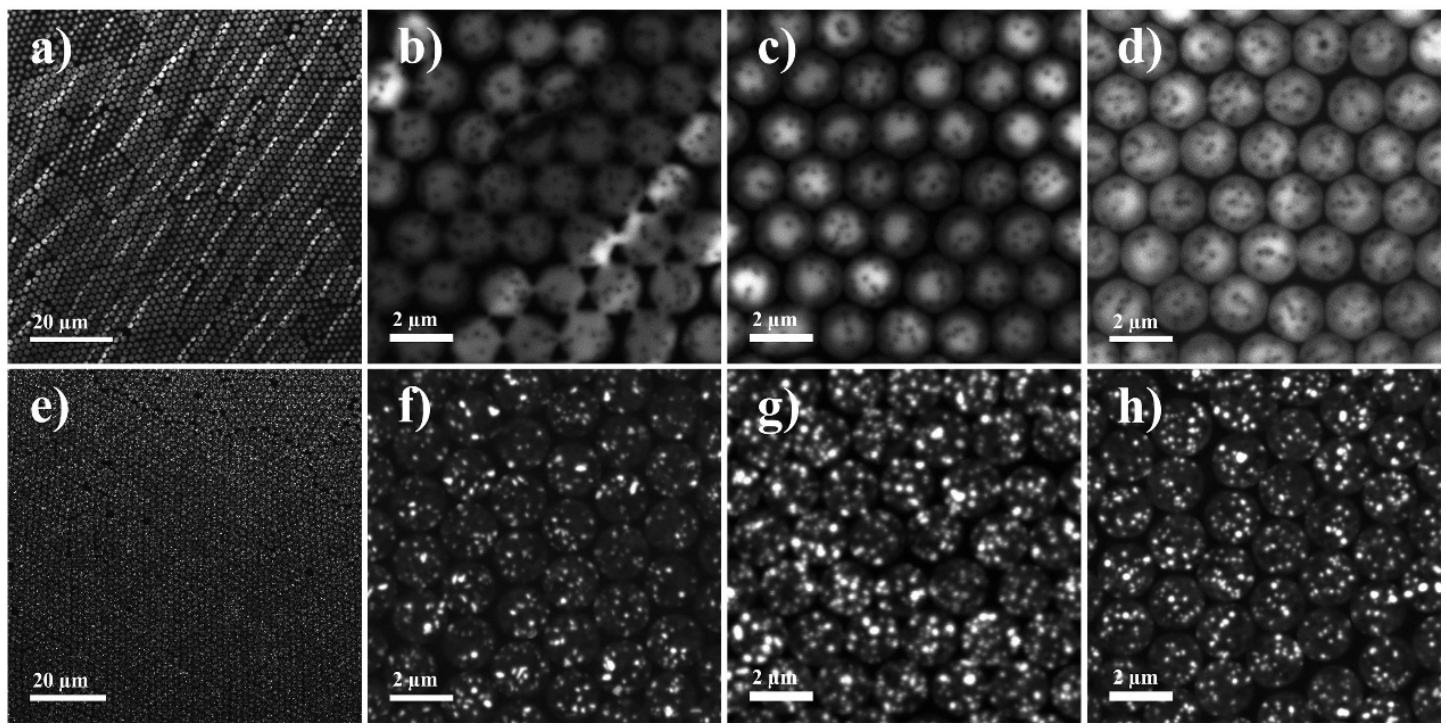

Figure 6. Low magnification (a) and high magnification panchromatic cathodoluminescence (CL) mapping of as-fabricated (b), $\mathrm{KOH}$ treated (c) and thermally treated (d) III-polar nanopillar MQWs. CL mapping of N-polar nanopillar MQWs are illustrated in (e-h), accordingly. 

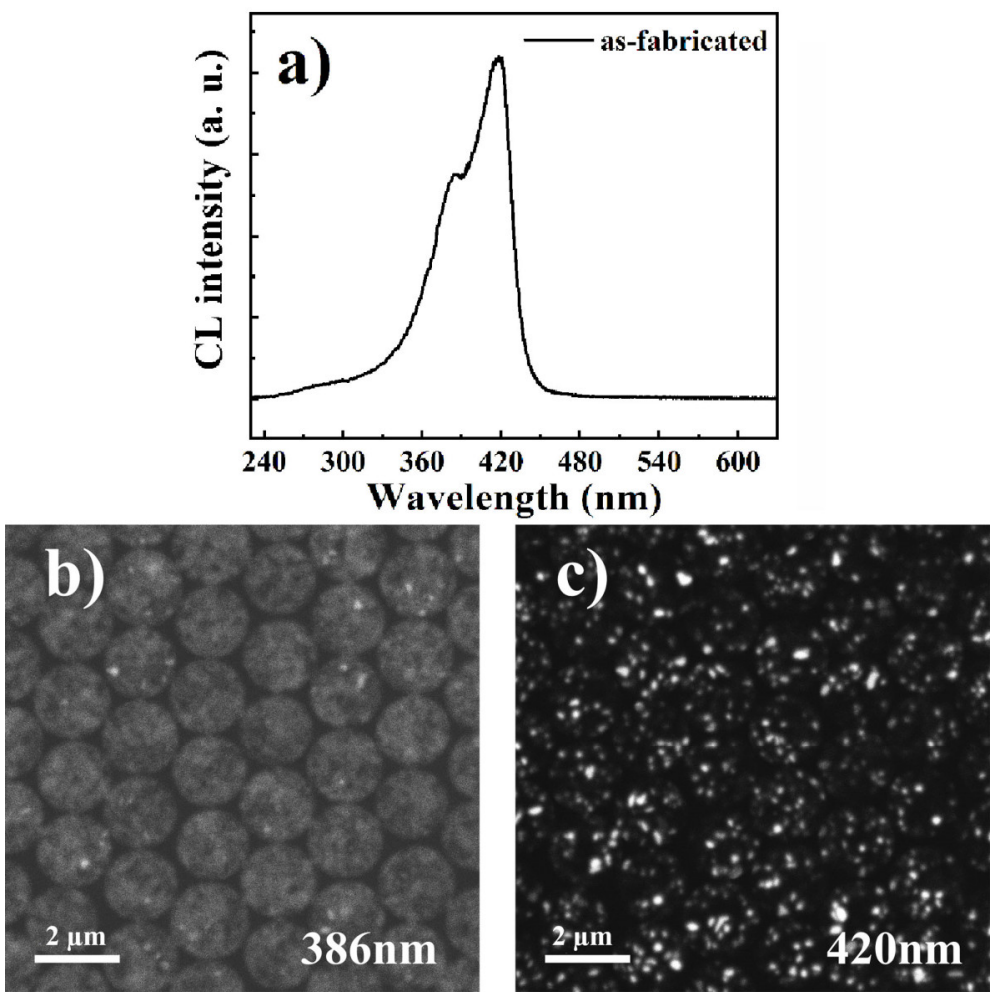

Figure 7. CL spectrum of N-polar nanopillar MQW illustrating two peaks at 386 and $420 \mathrm{~nm}$, respectively (a). Monochromatic CL intensity distribution of N-polar nanopillar MQW at $386 \mathrm{~nm}$ (b) and $420 \mathrm{~nm}$ (c), respectively.

Additionally, the black dots were identified throughout the surface in the panchromatic CL intensity mapping shown in Figure $6 \mathrm{~b}-\mathrm{d}$, representing non-radiative recombination centers like threading dislocations or point defects. The densities were $2.35 \times 10^{8} \mathrm{~cm}^{2}, 2.29 \times 10^{8} \mathrm{~cm}^{2}$ and $2.38 \times 10^{8} \mathrm{~cm}^{2}$ respectively for as-fabricated nanopillars, nanopillars subject to $\mathrm{KOH}$ treatment and thermal annealing, respectively. No obvious change in the defect density after thermal or $\mathrm{KOH}$ treatment was identified, suggesting that luminescence enhancement as shown in Figure 4 is mainly related to passivation of surface states, rather than defects in the bulk.

Figure 7a shows the CL spectrum of the as-fabricated N-polar nanopillars with two peaks located at 386 and $420 \mathrm{~nm}$. The monochromatic CL mapping at $386 \mathrm{~nm}$ and $420 \mathrm{~nm}$ are shown in Figure 7b,c, respectively. Interestingly, the CL emission at $386 \mathrm{~nm}$ was uniformly distributed on the surface of the nanopillars, whereas $420 \mathrm{~nm}$-emission originated from In-rich clusters, which are represented by the white dots. The red shift of CL spectra for N-polar nanopillars compared to that of the III-polar sample can be well explained by the in-phase separation in the MQWs leading to quantum dot-like luminescence centers at those In-rich clusters. Additionally, the carrier diffusion length decreased drastically due to indium phase separation, and the influence of point defects and threading dislocations were mostly screened by these potential minima, imposing no effect on the luminescence intensity. Therefore, even though surface trap defects were passivated by thermal and chemical treatments the luminescence intensities still remained the same for N-polar nanopillar MQWs because of carrier localization effects.

Optical properties of MQWs were also correlated to strain conditions. Therefore, Raman spectroscopy investigations were performed. Figure 8 shows the Raman spectra recorded for IIIand N-polar nanopillars before and after RTA or $\mathrm{KOH}$ treatment. The peak position of the $\mathrm{E}_{2}$ (high) phonon mode of III-nitride crystal is sensitive to bi-axial strains [30]. For fully relaxed GaN, the $\mathrm{E}_{2}$ (high) peak was at $567.6 \mathrm{~cm}^{-1}$, and a smaller Raman wavenumber suggests tensile strain while a larger Raman wavenumber suggests the compressive strain. In this work, since the epitaxial thin film was 
mainly composed of $\mathrm{GaN}$ or $\mathrm{AlGaN}$ with low $\mathrm{Al}$ composition, $\mathrm{E}_{2}$ (high) peak located around $567 \mathrm{~cm}^{-1}$ was regarded as fully-relaxed, and was utilized for strain analysis. The peak positions for all the III-polar nanopillar MQW samples were close to each other with a value of $569 \mathrm{~cm}^{-1}$ indicating slightly compressive strains of $0.6 \mathrm{Gpa}$ regardless of chemical or thermal treatment. Note that the $\mathrm{E}_{2}$ (high) position of the as-fabricated planar MQW was located at a much high wavenumber of $572.1 \mathrm{~cm}^{-1}$ as observed in our recent study. Therefore, most of the strains were actually relaxed after nanopillar fabrication. Compared to the III-polar nanopillar samples, the compressive strain in as-fabricated $\mathrm{N}$-polar nanopillar samples was found to be much smaller as the $\mathrm{E}_{2}$ (high) position of the GaN-like Raman peak was only $1 \mathrm{~cm}^{-1}$ higher at maximum compared to the fully relaxed position. This could be explained by the 3D growth mode of $\mathrm{N}$-polar thin film, higher dislocation density and thus more strain relaxation inside $\mathrm{N}$-polar crystals. RTA treatment at $900^{\circ} \mathrm{C}$ fully relaxes the compressive strain inside the as-fabricated N-polar nanopillars, even though no significant improvement in emission intensity was found.
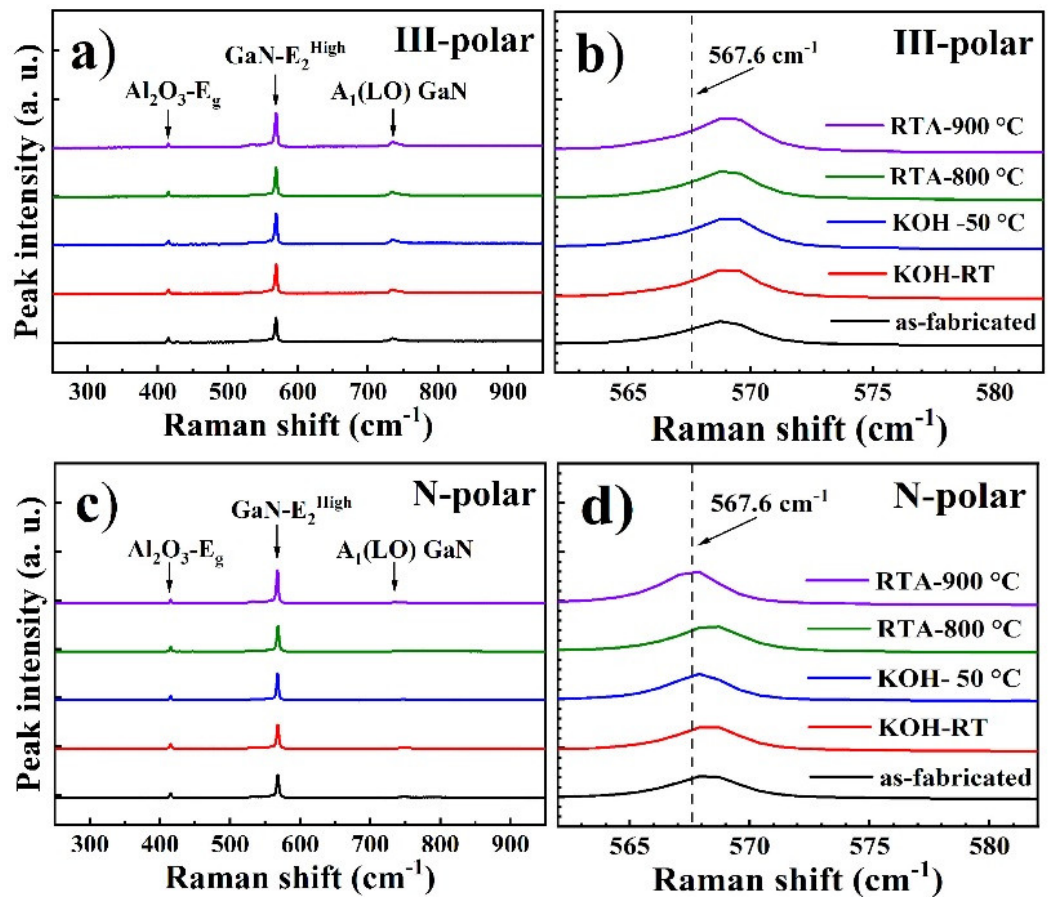

Figure 8. Full-range (a,c) and zoom-in (b,d) Raman spectra of III-polar (a,b) and N-polar (c,d) nanopillar MQWs before and after chemical and thermal treatments.

Finally, to further correlate surface stoichiometry to the enhanced UV emission of III-polar nanopillar MQWs, XPS measurements were carried out before and after RTA or KOH treatment. The de-convoluted $\mathrm{Ga} 3 \mathrm{~d}$ peaks of the as-fabricated nanopillars, nanopillars after $\mathrm{KOH}$ treatment and $900{ }^{\circ} \mathrm{C}$ annealing are shown in Figure 9a. As indicated in the figure, the major Ga 3d core level peak consisted of two components at $21.0 \pm 0.1 \mathrm{eV}$ and $19.3 \pm 0.1 \mathrm{eV}$ corresponding to the binding energy of $\mathrm{Ga}-\mathrm{O}$ and $\mathrm{Ga}-\mathrm{N}$, respectively. After $\mathrm{KOH}$ etching, the $\mathrm{Ga}$ 3d peak slightly shifted to higher binding energy, where the relative $\mathrm{Ga}-\mathrm{O}$ component was increased, indicating that $\mathrm{Ga}$ cations were attacked by $\mathrm{OH}-$, leading to the breakage of $\mathrm{Ga}-\mathrm{N}$ bond and consequently formation of $\mathrm{Ga}-\mathrm{O}$ bond. Furthermore, after RTA surface treatment, the Ga $3 \mathrm{~d}$ peak shifts to even higher binding energy, but the total intensity was dramatically reduced. This indicates that chemical stoichiometry between $\mathrm{Ga}$ and $\mathrm{N}$ atom was deviating from 1:1 after thermal annealing. Figure $9 \mathrm{~b}$ shows the $\mathrm{O} 1 \mathrm{~s}$ core level peaks de-convoluted into three components with binding energies of $530.3 \mathrm{eV}, 531.7 \mathrm{eV}$ and $532.8 \mathrm{eV}$, corresponding to $\mathrm{O}^{2-}$, $\mathrm{OH}^{-}$and $\mathrm{H}_{2} \mathrm{O}$ species, respectively. It is clearly shown that after $\mathrm{KOH}$ etching, the overall intensity of the $\mathrm{O}$ 1s peak decreased, in agreement with the removal of naturally formed surface oxide under $\mathrm{KOH}$ 
etching. After thermal annealing, there existed a strong shift of $\mathrm{O}$ 1s peak towards higher binding energy, indicating a transformation from the $\mathrm{Ga}-\mathrm{O}$ bond to $\mathrm{Ga}-\mathrm{O}-\mathrm{H}$ bond.
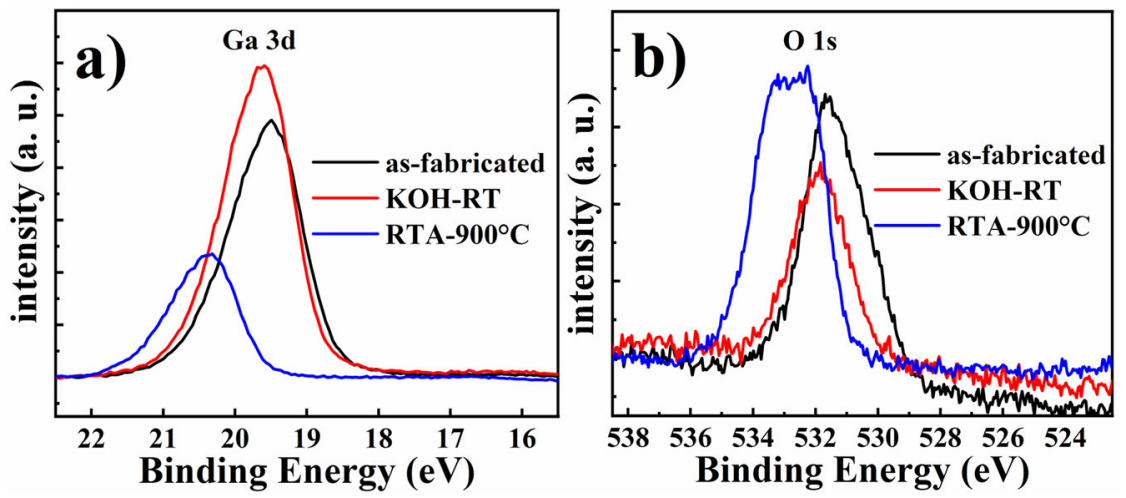

Figure 9. High resolution Ga 3d (a) and O 1s (b) XPS core level spectra of III-polar nanopillar MQWs without treatment and after $\mathrm{KOH}$ or RTA treatments.

\section{Conclusions}

In this work, the influences of $\mathrm{KOH}$ or RTA treatments on the optical properties of III and N-polar nanopillar UV MQWs were carefully examined. The UV emission intensity increased significantly after post-fabrication treatment and by as much as 2.4 times after RTA annealing for III-polar nanopillars, whereas almost no effect on the luminescence spectra was seen for N-polar samples. CL intensity maps indicate carrier localization effects due to In-rich clusters in the N-polar nanopillars, which dominated the light output mechanism because of the reduced carrier diffusion length scales. This led to reduced probability of carrier recombination with threading dislocations or point defects, meaning that despite the fact that surface treatment passivation methods might reduce the trap defects, they did not contribute to the PL efficiency. Slight compressive strain effects could be found in as-fabricated III-polar nanopillars, but which did not change significantly after $\mathrm{KOH}$ or RTA treatment. As-fabricated N-polar nanopillar samples were almost strain-free due to 3D growth mode and strain relaxation mechanism. XPS results further suggest the removal of surface oxides after $\mathrm{KOH}$ etching and transformation from $\mathrm{Ga}-\mathrm{O}$ to $\mathrm{Ga}-\mathrm{O}-\mathrm{H}$ bond after thermal annealing for III-polar nanopillar samples. The experimental results obtained here present a deeper understanding on the different optical behaviors of nanostructured UV emitters with opposite polarities, and provide an important strategy to enhance the luminescence intensity of nanopillar LEDs, paving the way for future development of novel nanostructure-based electronic or photonic devices.

Supplementary Materials: The following are available online at http://www.mdpi.com/2072-666X/11/6/572/s1, Figure S1: HRXRD symmetric $\omega-2 \theta$ scans of III- and N-polar MQW samples; Figure S2: RT PL spectra of planar III-polar and N-polar MQW samples. Peak positions and shapes are almost identical to those of nanostructured samples; Figure S3: Simulated electroluminescence spectrum of UVA-LED with 3/11 nm $\operatorname{In}_{0.03} \mathrm{Ga}_{0.97} \mathrm{~N} / \mathrm{GaN} \mathrm{MQW}$; Figure S4: HAADF-STEM image of planar III-polar MQW samples with low magnification (a) and zoom-in view (b) of the individual QW and QB; Figure S5: Simulated electroluminescence spectra of UVA-LED with $\mathrm{In}_{0.03} \mathrm{Ga}_{0.97} \mathrm{~N} / \mathrm{GaN}$ MQWs of varying QW thicknesses. A small red shift of less than $7 \mathrm{~nm}$ is observed when doubling the QW thickness.

Author Contributions: Data curation, M.S., Y.D. and M.C.; Formal analysis, L.L., J.L., W.L. and R.J.; Supervision, K.W.A.C. and J.Y.; Writing-original draft, W.G. All authors have read and agreed to the published version of the manuscript.

Funding: This work is supported by National Key Research and Development Program of China (Grant 2016YFB0400802), National Natural Science Foundation of China (Grants 61974149, 61704176, 61950410617 and 61874177), Key Research and Development Program of Zhejiang Province (2020C01145).

Conflicts of Interest: The authors declare no conflict of interest. 


\section{References}

1. Kneissl, M.; Seong, T.-Y.; Han, J.; Amano, H. The emergence and prospects of deep-ultraviolet light-emitting diode technologies. Nat. Photonics 2019, 13, 233. [CrossRef]

2. Hirayama, H.; Maeda, N.; Fujikawa, S.; Toyoda, S.; Kamata, N. Recent progress and future prospects of AlGaN-based high-efficiency deep-ultraviolet light-emitting diodes. Jpn. J. Appl. Phys. 2014, 53, 100209. [CrossRef]

3. Xu, H.; Sheikhi, M.; Li, L.; Yang, Z.; Hoo, J.; Guo, S.; Zeng, Y.; Guo, W.; Ye, J. Omnidirectional whispering-gallery-mode lasing in GaN microdisk obtained by selective area growth on sapphire substrate. Opt. Express 2019, 27, 16195-16205.

4. Guo, W.; Mitra, S.; Xu, H.; Sheikhi, M.; Sun, H.; Tian, K.; Zhang, Z.-H.; Jiang, H.; Roqan, I.S.; Li, X. Three-dimensional band diagram in lateral polarity junction III-nitride heterostructures. Optica 2019, 6, 1058-1062. [CrossRef]

5. Xu, H.; Long, H.; Sheikhi, M.; Li, L.; Guo, W.; Dai, J.; Chen, C.; Ye, J. Strain modulated nanostructure patterned AlGaN-based deep ultraviolet multiple-quantum-wells for polarization control and light extraction efficiency enhancement. Nanotechnology 2019, 30, 435202. [CrossRef] [PubMed]

6. Long, H.; Wang, S.; Dai, J.; Wu, F.; Zhang, J.; Chen, J.; Liang, R.; Feng, Z.C.; Chen, C. Internal strain induced significant enhancement of deep ultraviolet light extraction efficiency for AlGaN multiple quantum wells grown by MOCVD. Opt. Express 2018, 26, 680-686. [CrossRef] [PubMed]

7. Guo, W.; Yang, Z.; Li, J.; Yang, X.; Zhang, Y.; Wang, J.; Chee, K.; Gao, P.; Ye, J. Enhancing light coupling and emission efficiencies of $\mathrm{AlGaN}$ thin film and $\mathrm{AlGaN} / \mathrm{GaN}$ multiple quantum wells with periodicity-wavelength matched nanostructure array. Nanoscale 2017, 9, 15477. [CrossRef] [PubMed]

8. Mandl, M.; Wang, X.; Schimpke, T.; Kölper, C.; Binder, M.; Ledig, J.; Waag, A.; Kong, X.; Trampert, A.; Bertram, F. Group III nitride core-shell nano-and microrods for optoelectronic applications. Phys. Status Solidi RRL Rapid Res. Lett. 2013, 7, 800-814. [CrossRef]

9. Le, B.H.; Zhao, S.; Liu, X.; Woo, S.Y.; Botton, G.A.; Mi, Z. Controlled coalescence of AlGaN nanowire arrays: An architecture for nearly dislocation-free planar ultraviolet photonic device applications. Adv. Mater. 2016, 28, 8446-8454. [CrossRef]

10. Zhang, L.; Guo, Y.; Yan, J.; Wu, Q.; Lu, Y.; Wu, Z.; Gu, W.; Wei, X.; Wang, J.; Li, J. Deep ultraviolet light-emitting diodes based on a well-ordered AlGaN nanorod array. Photonics Res. 2019, 7, B66-B72. [CrossRef]

11. Maeda, N.; Jo, M.; Hirayama, H. Improving the light-extraction efficiency of AlGaN DUV-LEDs by using a superlattice hole spreading layer and an Al reflector. Phys. Status Solidi a Appl. Mater. Sci. 2018, 215, 1700436. [CrossRef]

12. Zhang, Z.-H.; Kou, J.; Chen, S.-W.H.; Shao, H.; Che, J.; Chu, C.; Tian, K.; Zhang, Y.; Bi, W.; Kuo, H.-C. Increasing the hole energy by grading the alloy composition of the p-type electron blocking layer for very high-performance deep ultraviolet light-emitting diodes. Photonics Res. 2019, 7, B1-B6. [CrossRef]

13. Li, Q.; Westlake, K.R.; Crawford, M.H.; Lee, S.R.; Koleske, D.D.; Figiel, J.J.; Cross, K.C.; Fathololoumi, S.; Mi, Z.; Wang, G.T. Optical performance of top-down fabricated InGaN/GaN nanorod light emitting diode arrays. Opt. Express 2011, 19, 25528-25534. [CrossRef] [PubMed]

14. Guo, W.; Li, J.; Sheikhi, M.; Jiang, J.; Yang, Z.; Li, H.; Guo, S.; Sheng, J.; Sun, J.; Bo, B. Comparative study on luminescence extraction strategies of LED by large-scale fabrication of nanopillar and nanohole structures. J. Phys. D Appl. Phys. 2018, 51, 24LT01. [CrossRef]

15. Wang, Q.; Zhao, S.; Connie, A.; Shih, I.; Mi, Z.; Gonzalez, T.; Andrews, M.; Du, X.; Lin, J.; Jiang, H. Optical properties of strain-free AlN nanowires grown by molecular beam epitaxy on Si substrates. Appl. Phys. Lett. 2014, 104, 223107. [CrossRef]

16. Hartensveld, M.; Ouin, G.; Liu, C.; Zhang, J. Effect of $\mathrm{KOH}$ passivation for top-down fabricated InGaN nanowire light emitting diodes. J. Appl. Phys. 2019, 126, 183102. [CrossRef]

17. Sheikhi, M.; Guo, W.; Dai, Y.; Cui, M.; Hoo, J.; Guo, S.; Xu, L.; Liu, J.; Ye, J. Mechanism of improved luminescence intensity of Ultraviolet Light Emitting Diodes (UV-LEDs) under thermal and chemical treatments. IEEE Photonics J. 2019. [CrossRef]

18. Chee, K.W.; Guo, W.; Wang, J.R.; Wang, Y.; Chen, Y.-E.; Ye, J. Tuning photonic crystal fabrication by nanosphere lithography and surface treatment of AlGaN-based ultraviolet light-emitting diodes. Mater. Des. 2018, 160, 661-670. [CrossRef] 
19. Chiu, C.; Lu, T.-C.; Huang, H.; Lai, C.; Kao, C.; Chu, J.; Yu, C.; Kuo, H.-C.; Wang, S.; Lin, C. Fabrication of InGaN/GaN nanorod light-emitting diodes with self-assembled Ni metal islands. Nanotechnology 2007, 18, 445201. [CrossRef]

20. Guo, W.; Sun, H.; Torre, B.; Li, J.; Sheikhi, M.; Jiang, J.; Li, H.; Guo, S.; Li, K.H.; Lin, R. Lateral-polarity structure of AlGaN quantum wells: A promising approach to enhancing the ultraviolet luminescence. Adv. Funct. Mater. 2018, 28, 1802395. [CrossRef]

21. Keller, S.; Li, H.; Laurent, M.; Hu, Y.; Pfaff, N.; Lu, J.; Brown, D.; Fichtenbaum, N.; Speck, J.; DenBaars, S.; et al. Recent progress in metal-organic chemical vapor deposition of N-polar group-III nitrides. Semicond. Sci. Technol. 2014, 29, 113001. [CrossRef]

22. Bryan, I.; Bryan, Z.; Mita, S.; Rice, A.; Hussey, L.; Shelton, C.; Tweedie, J.; Maria, J.; Collazo, R.; Sitar, Z. The role of surface kinetics on composition and quality of AlGaN. J. Cryst. Growth 2016, 451, 65-71. [CrossRef]

23. Sun, Q.; Cho, Y.; Lee, I.; Han, J.; Kong, B.; Cho, H. Nitrogen-polar GaN growth evolution on c-plane sapphire. Appl. Phys. Lett. 2008, 93, 131912. [CrossRef]

24. Chichibu, S.; Sota, T.; Wada, K.; Brandt, O.; Ploog, K.; DenBaars, S.; Nakamura, S. Impact of internal electric field and localization effect on quantum well excitons in AlGaN/GaN/InGaN light emitting diodes. Phys. Status Solidi (A) 2001, 183, 91-98. [CrossRef]

25. Sánchez-Rojas, J.; Garrido, J.; Munoz, E. Tailoring of internal fields in AlGaN/GaN and InGaN/GaN heterostructure devices. Phys. Rev. B 2000, 61, 2773. [CrossRef]

26. Tan, S.; Zhang, J.; Egawa, T.; Chen, G.; Luo, X.; Sun, L.; Zhu, Y. Influence of quantum-well width on the electroluminescence properties of AlGaN deep ultraviolet light-emitting diodes at different temperatures. Nanoscale Res. Lett. 2018, 13, 1-5. [CrossRef] [PubMed]

27. Cho, Y.; Gainer, G.; Fischer, A.; Song, J. "S-shaped" temperature-dependent emission shift and carrier dynamics in InGaN/GaN multiple quantum wells. Appl. Phys. Lett. 1998, 73, 1370-1372. [CrossRef]

28. Liu, W.; Zhao, D.; Jiang, D.; Shi, D.; Zhu, J.; Liu, Z.; Chen, P.; Yang, J.; Liang, F.; Liu, S.; et al. Effect of carrier transfer process between two kinds of localized potential traps on the spectral properties of InGaN/GaN multiple quantum wells. Opt. Express 2018, 26, 3427-3434. [CrossRef]

29. Lin, Y.; Ma, K.; Hsu, C. Dependence of composition fluctuation on indium content in InGaN/GaN multiple quantum wells. Appl. Phys. Lett. 2000, 77, 2988-2990. [CrossRef]

30. Perlin, P.; Jauberthie-Carillon, C.; Itie, J.P.; Miguel, A.S.; Grzegory, I.I.; Polian, A. Raman scattering and $\mathrm{x}$-ray-absorption spectroscopy in gallium nitride under high pressure. Phys. Rev. B 1992, 45, 83-89. [CrossRef]

(C) 2020 by the authors. Licensee MDPI, Basel, Switzerland. This article is an open access article distributed under the terms and conditions of the Creative Commons Attribution (CC BY) license (http://creativecommons.org/licenses/by/4.0/). 